\title{
Virtual autopsy and confirmation of normal fetal heart anatomy in the first trimester using three-dimensional (3D) reconstruction of histological sections
}

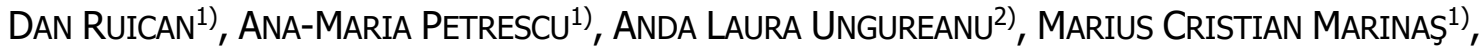

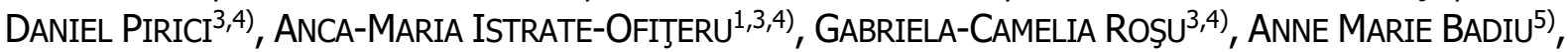

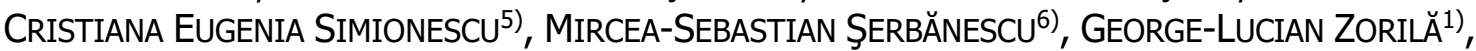 \\ SMARANDA BELCIUG ${ }^{6}$, DOMINIC-GABRIEL ILIESCU ${ }^{1,7,8)}$
}

\author{
1) Department of Obstetrics and Gynecology, Emergency County Hospital, Craiova, Romania \\ 2) Department of Cardiology, University of Medicine and Pharmacy of Craiova, Romania \\ ${ }^{3)}$ Department of Histology, University of Medicine and Pharmacy of Craiova, Romania \\ 4) Research Center for Microscopic Morphology and Immunology, University of Medicine and Pharmacy \\ of Craiova, Romania \\ 5) Department of Pathology, Emergency County Hospital, Craiova, Romania \\ 6) Department of Medical Informatics and Biostatistics, University of Medicine and Pharmacy of Craiova, \\ Romania \\ 7) Department of Mother and Child, University of Medicine and Pharmacy of Craiova, Romania \\ 8) Department of Maternal-Fetal Medicine, MEDGIN GINECHO Clinic, Craiova, Romania
}

\begin{abstract}
Objective: In this pilot study, we tested the feasibility of cardiac structures reconstruction from histological sections in 12-13 weeks normal fetuses. Conventional autopsy is hampered at this gestational age because of the small size of the heart anatomical structures, while alternative non-invasive methods for pathology examination of the fetus are expensive, rarely available and lack accuracy data regarding the confirmation of first trimester heart defects suspected by early prenatal ultrasound (US) scans. Materials and Methods: Normal hearts from fetuses aged 12-13 gestational weeks (GW) were harvested for histological preparation, virtual reconstruction, and cardiac structures analysis. The normalcy of heart structures was confirmed before pregnancy termination, using a detailed US scan protocol. The fetal heart was routinely processed for formalin fixation and paraffin embedding (FFPE) and $10 \mu \mathrm{m}$ seriate sections have been cut until finishing the specimen. All sections have been scanned and a three-dimensional (3D) reconstruction of the whole organ has been rendered, based on computer-aided manual tracing. Using the 3D navigation software, the main cardiac structures were searched for a proper and confident visualization. Results: Five cases were investigated. Visualization of the normal heart cavities, including atrioventricular septum was very good in all fetuses. The entire course of right and left ventricle outflow tracts was confidently confirmed, along the branching pattern of aorta and pulmonary artery trunk. Regarding the veno-atrial connections, it was easy to identify the entrance of the inferior and superior caval veins into the right atrium, but a detailed review of the histological sections was necessary for the visualization of the left atrium venous openings. The inherent morphological deformation following heart block sectioning resulted in a lower resolution or quality of the "reconstructed" planes, but these distortions did not represent a significant impediment in any of the cases. The resources involved ordinary histology and information technology (IT) equipment. To further decrease the time involved by the protocol, many steps may be automated: cutting, coloring, and scanning. Conclusions: The results indicate that this method can be implemented to routine clinical practice. The use of 3D reconstruction of fetal heart histological sections in first trimester may serve as an important audit to confirm the normalcy of heart structures. Also, the histological and postprocessed information is retained, and this volume can be stored, reanalyzed, or sent online for a second opinion. The method involves relatively undemanding resources, i.e., hardware, software, competences, and time. The procedure could also benefit from refinements used in other imaging techniques to limit human-computer interactions, such as sections distortion.
\end{abstract}

Keywords: virtual fetal anatomy, prenatal diagnosis, perinatal autopsy, histology, prenatal ultrasound.

\section{Introduction}

Fetal medicine is improving hastily due to technological expansion combined with rising diagnostic experience.

The evolution of prenatal diagnosis enabled the detection of fetal structural abnormalities as early as the end of the first trimester of pregnancy. The first trimester anomaly scan became part of the routine pregnancy care [1], and specific recommendations have been developed by professional societies regarding the objectives and technique of the ultrasound (US) scan [2]. Still, the termination of pregnancy due to fetal severe malformations should be audited according to an accurate anatomic diagnosis, to confirm the echocardiographic diagnosis that prompted the pregnancy termination. Frequently, fetal malformations at this early gestational age are highlighted by using indirect

This is an open-access article distributed under the terms of a Creative Commons Attribution-NonCommercial-ShareAlike 4.0 International Public License, which permits unrestricted use, adaptation, distribution and reproduction in any medium, non-commercially, provided the new creations are licensed under identical terms as the original work and the original work is properly cited. 
techniques, as the Doppler imaging of the functional heart activity. Conventional autopsy remains the "gold standard" for fetal death or stillbirth evaluation [3], and the confirmation of fetal defects suspected at the prenatal US evaluations. Several large studies found that autopsy added significant information regarding fetal heart in up to one quarter of cases, when compared to prenatal US diagnosis $[4,5]$ at a second trimester average gestational age, which offer a better view on fetal heart structures than first trimester assessment. Moreover, in first trimester fetuses, an accurate conventional autopsy is hampered by major difficulties, due to the small dimensions of the fetal heart, accompanied by an equal complexity of its anatomy and the wide diversity of cardiac malformation.

Ex vivo non-invasive imaging techniques, such as highresolution 7-9.4 T magnetic resonance imaging (MRI) [6-8] and microfocus computed tomography (micro-CT) $[9,10]$, were proposed as alternatives for autopsy in small fetuses, less than 22 gestational weeks $(\mathrm{GW})$, where the information provided by conventional 1.5-3 T MRI has important limitations [11-13]. Still, we have only limited data regarding the detection of heart anomalies in first trimester fetuses and the equipment's and expert's availability is very low.

Advanced pathology procedures include dissection microscopy and episcopic microscopy. Dissection microscope was frequently used to confirm the anatomy in small fetuses, but this requires special instrumentation and medical training. Episcopic microscopy $[14,15]$, represents one of the most accurate confirmation methods. The episcopic alignment of images prevents distortions and excludes realignment difficulties [16, 17], but is rarely performed, due to the resources involved, the difficulties in obtaining a balanced and homogeneous staining of the sample [18] and lacks the benefit to reanalyze the original sectioned tissues.

One of the first methods used to imagine embryonic developing structures was three-dimensional (3D) reconstructions of histological sections based on computerassisted manual delimitation of the organs of interest, illustrated by medical artists or dedicated software $[16,19,20]$. A previous pilot study confirmed that the improvement of 3D reconstruction methods enables accurate visualization and measurement of internal and external cardiac structures and large vessels in fetuses younger than $13 \mathrm{GW}$ [21]. Nevertheless, this technique was considered difficult and labor-intensive as it required sophisticated manual cutting, histological preparation and alignment of many sections and therefore left only for researcher's purposes. Still, nowadays many of these procedures have been automatized.

\section{Aim}

The aim of our pilot study was to describe an easily reproducible protocol meant to reconstruct fetal heart anatomy using serial histological sections. This method could be used widely in fetal medicine centers as an audit for the first trimester heart scan, in pregnancy terminations and miscarriages cases.

\section{a Materials and Methods}

The study protocol was proposed to pregnant woman admitted in our Clinic for pregnancy termination at request in the end of the first trimester (12-14 GW). Medical abortion protocol was elected, that involved Mifepristone in conjunction with Misoprostol. The parents were informed regarding our research project and agreed with the autopsy and histopathological (HP) examination presented in this paper.

Fetal anatomy was carefully evaluated before termination by a team of experts in maternal-fetal medicine and pediatric cardiology, according to a detailed first trimester protocol, using both transabdominal and transvaginal approach. The US scans were performed using Philips EPIQ 7 system, Philips Ultrasound Inc., Bothell, USA, equipped with the eL18-4 linear array, V9-2, 3D9-3v convex 3D array and General Electric Voluson E10 system, GE Healthcare, Zipf, Austria, equipped with RM6C and RIC6-12 convex transducers.

Fetal autopsy was performed following the evacuation of the fetuses in the Department of Pathology, Emergency County Hospital, Craiova, where, during the procedure, the fetal hearts were harvested and sent for further evaluation at the Research Center for Microscopic Morphology and Immunology, University of Medicine and Pharmacy of Craiova, Romania. The whole tissue was fixed in $10 \%$ neutral buffered formalin and routinely processed for formalin fixation and paraffin embedding (FFPE). The block was then sectioned in seriate $10 \mu \mathrm{m}$ thick sections until no tissue was left visible, using a motorized HMB450 rotary microtome (Thermo Scientific), using constant speed and strokes. Sectioned slices were collected utilizing a dedicated section transfer system (STS), flattened on a warm water bath $\left(37^{\circ} \mathrm{C}\right)$, collected on poly-L-lysine treated slides for better adhesion, and left to dry in a thermostat $\left(37^{\circ} \mathrm{C}\right)$ for 24 hours. The slides were all numbered to keep the slices in the correct order and 300 slices were created. Subsequently, we used the protocols for the classical histological technique for Hematoxylin-Eosin (HE). The slides were deparaffinized, rehydrated with decreasing concentrations of ethanol, till distilled water. After rehydration, the sectioned were stained with HE, all batches of slides being processed with exact timings. The sections were last coverslipped with a xylene-based mounting medium.

The slides were scanned utilizing a Nikon Eclipse 90i motorized microscope (Nikon Instruments, Europe BV, Amsterdam, The Netherlands) fitted with a Prior ES111 OptiScan motorized stage (Prior Scientific, Cambridge, UK), a 16-megapixel Nikon DS-Ri-2 complementary metal oxide semiconductor (CMOS) cooled camera and driven by the Nikon NIS-Elements AR image analysis software. Each image was numbered accordingly to maintain the order of the slices. The resulting images were processed using MATLAB algorithms (version 2019a, MathWorks, USA), which removed blood contained in the heart cavities, artefacts resulting from the slide scans. The canvas size of the images was adjusted to be the same for all [22]. At first, we undertook a preprocessing step that implied reducing the first image's size from $20 \mu \mathrm{m} /$ pixel to $400 \mu \mathrm{m} /$ pixel. The process is needed to reduce registration time. The staining process was not performed for the whole image set at the same time, resulting in different stain condition, thus different stain ratios on different slides. We have overcome this issue by applying a color transfer between images using a non-referenced version of the algorithm 
presented in [23]. The images were inverted and converted to an 8-bit grayscale, after which we have subtracted 10/256 points of intensity from each pixel to obtain a black uniform background. The pre-processing step ended with the transformation into a grayscale with clear background. The registration process involved using the current image as reference for its neighboring images. In the end, we have obtained a stack of 229 aligned images that were imported and processed in Amira-Avizo from ThermoFisher using a trial version. The slices were aligned using both automatic and manual alignment. Image segmentation was done manually by carefully selecting the anatomical elements (myocardium, aorta, pulmonary arteries, trachea, thymus, and the heart valves) one at a time on every slice using multiple selection tools that the software has to offer. Each individual element was attributed a "material". This made the selection and edit process much easier. The "blow" tool was first used to select the bulk of the elements using a tolerance of 20-35 and Gauss width of 4. The selection was then refined using the "brush" and "lasso" tool.

The automatic segmentation using the "threshold" tool did not yield the results we expected due to the presence of artefacts from the scanning of the slides and the processing of the images. However, it can be used to render the 3D volume but without the possibility of editing each heart element individually. The images underwent a smoothing process using the median filter. The settings used were the following: interpretation $3 \mathrm{D}$, neighborhood 26 , and the number of iterations were 5 . We found out that these settings were best for our model as further increasing of iterations would smooth the surface more, but heart cavities and vessel's lumen would fill up. The pictures were taken using the snapshot function, ".jpg" format, red, green, and blue (RGB) palette color.

The aim of the pathological assessment was to demonstrate the aspect of the cardiac chambers and atrioventricular (AV) septum, the venous atrial inflows and ventricular outflow tracts.

\section{ㅁ Results}

The multidisciplinary examination of the fetal anatomy was performed according to a detailed first trimester protocol (Figures 1 and 2). Both transabdominal and transvaginal techniques were used to investigate the first trimester fetal heart because of the complexity of the heart structures and small dimensions. The information provided by grayscale mode regarding heart normalcy was always complemented with color or power Doppler visualization of the blood flows, as the heart chambers and great vessels are usually too small for a proper visualization at this gestational age.
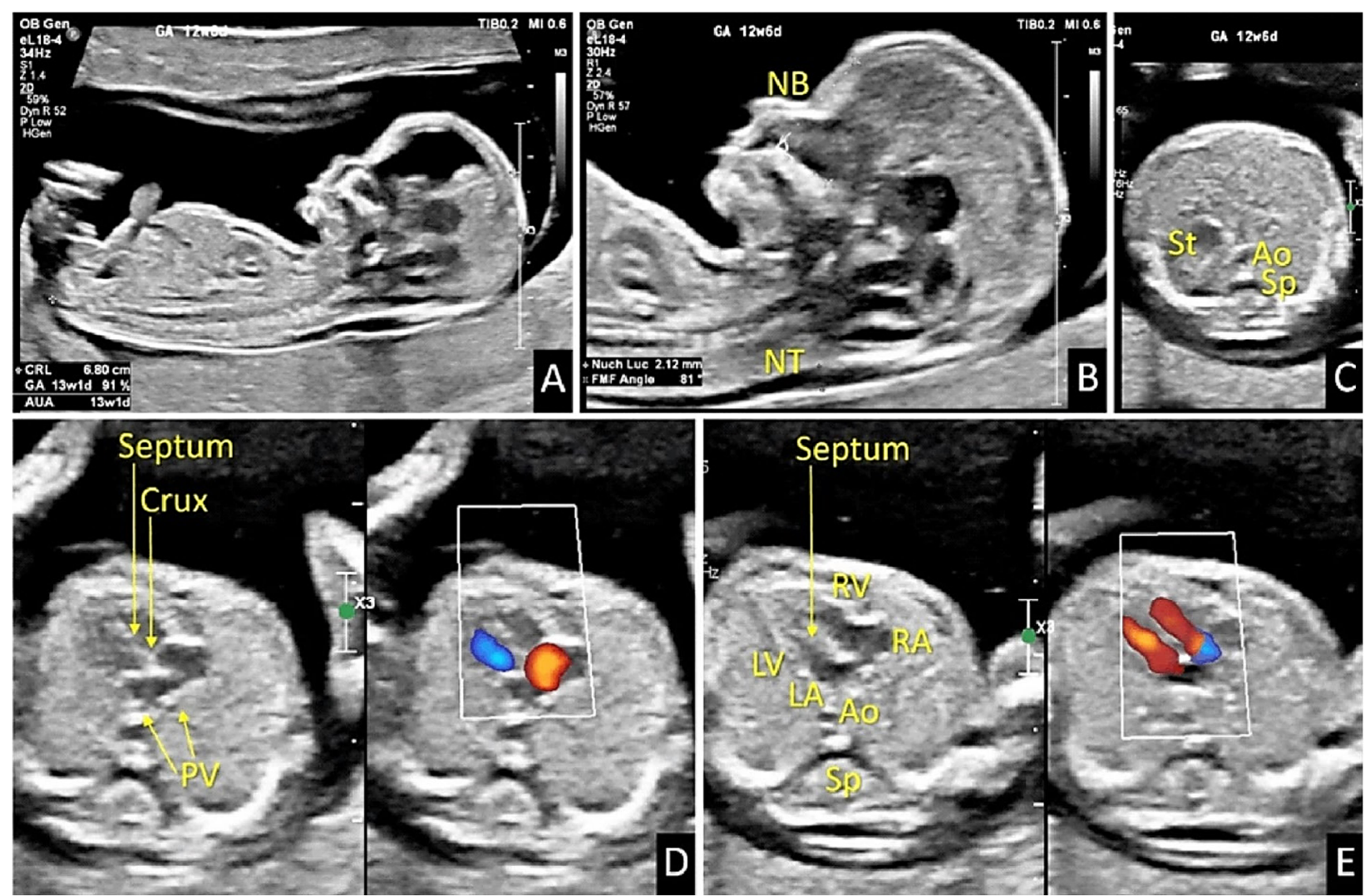

Figure 1 - Transabdominal evaluation of a 13+1 weeks' normal fetus at the time of the first trimester anomaly scan: (A) Crown-rump length measurement, to establish the gestational age; (B) Facial profile and evaluation of the genetic markers: nuchal translucency, nasal bone, and facial angle; (C) Situs evaluation in the axial upper abdominal plane at the beginning of cardiac sweep, with the stomach and aorta on the left side; (D) Cardiac sweep continuation with the visualization of the four-chamber plane during diastole in Compare mode (grayscale and high-definition power Doppler - Color Power Angio) showing cardiac chambers, septum and AV valves with crux cordis; (E) Four-chamber plane during systole in Compare mode showing opened mitral and tricuspid valves and equal and separate AV flows. Ao: Aorta; AV: Atrioventricular; LA: Left atrium; LV: Left ventricle; NB: Nasal bone; NT: Nuchal translucency; PV: Pulmonary veins; RA: Right atrium; RV: Right ventricle; Sp: Spine; St: Stomach. 

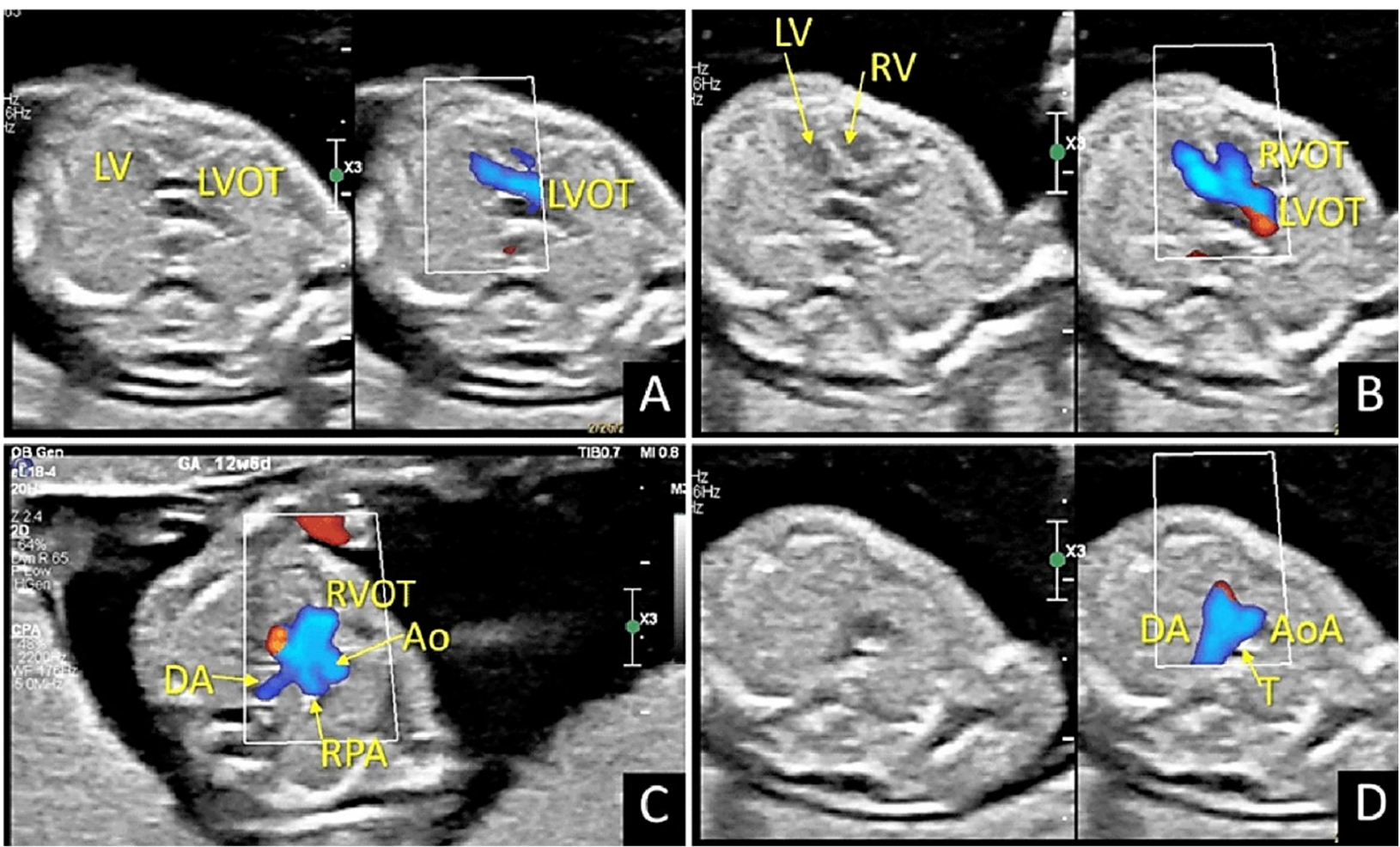

Figure 2 - Great vessels evaluation in the same fetus, during the cardiac sweep: (A) LVOT plane, in Compare mode with visualization of the aortic flow emerging from the $L V ;(B)$ Five-chamber plane in Compare mode, with aorta crossing the RVOT; (C) RVOT with pulmonary artery emerging from the $R V$ and branching in the axial plane; (D) Threevessel and trachea plane, duplex mode, showing the confluence of the arterial arches'flows using Doppler. Ao: Aorta; AoA: Aortic arch; DA: Ductal arch; LV: Left ventricle; LVOT: Left ventricular outflow tract; RPA: Right pulmonary artery; RV: Right ventricle; RVOT: Right ventricular outflow tract; T: Trachea.

After scanning the microscopic slides, we obtained a series of images that we analyzed and centralized in series (Figure 3A). Due to the nature of the HP preparation, some of the slices were more dilated than others and this aspect was corrected as well using MATLAB (Figure 3B). All images need different working tools in AmiraAvizo from ThermoFisher (Figure 3C).

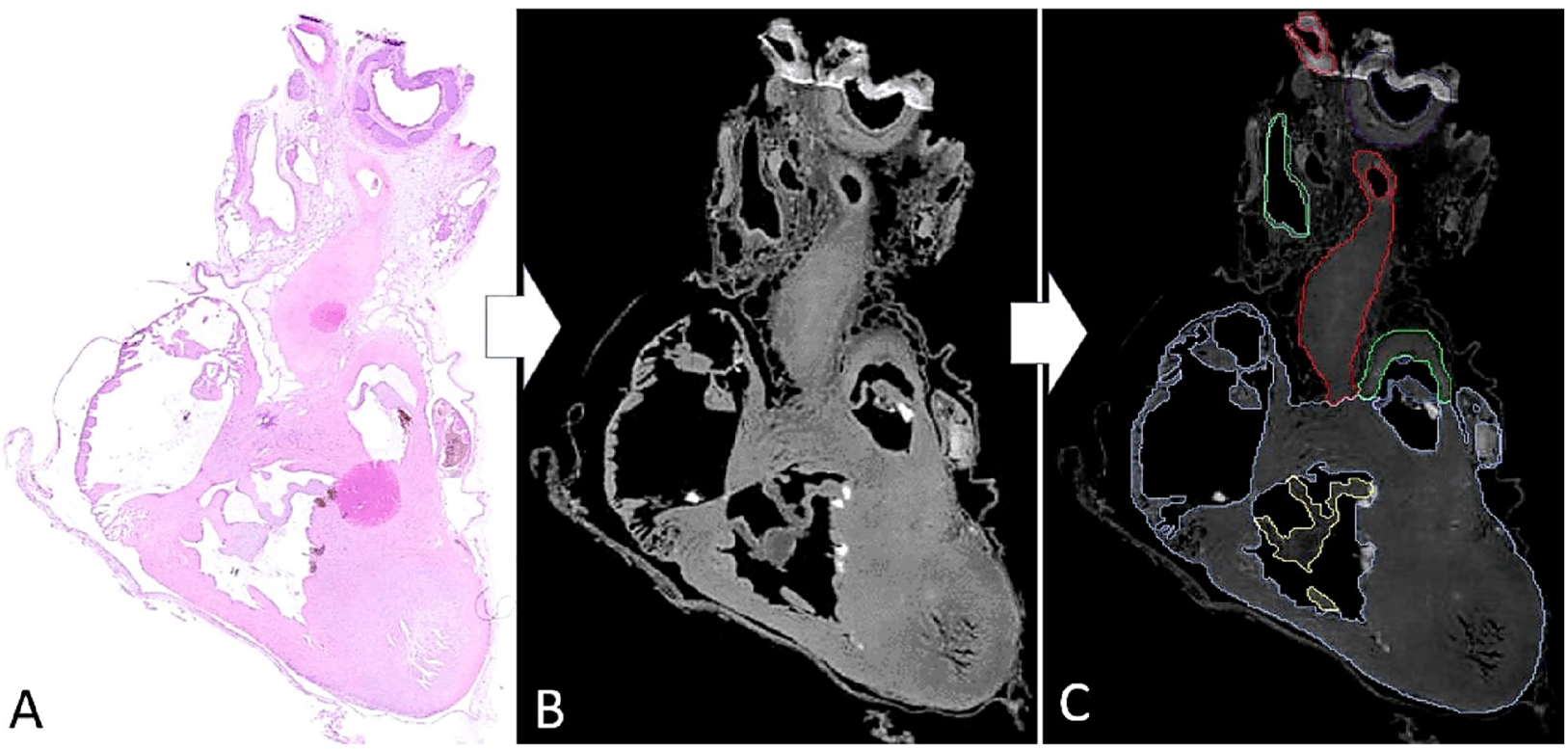

Figure 3 - (A) Histological capture of a heart section; (B) MATLAB post-processing image of the same capture; (C) Amira-Avizo segmentation editor view: individualizing each heart element.

A series of five fetal hearts harvested from $12+4$ to $13+2$ gestational age fetuses medically aborted were evaluated. Fetal heart measurements ranged between $14 / 5 / 3.5 \mathrm{~mm}$ and $17 / 7 / 4 \mathrm{~mm}$.
Visualization of the normal heart cavities, including AV septum was very good (Figures 4-6) in all fetuses. The emergence of right and left ventricle outflow tracts was also confidently identified, along their valves (Figures 4B 
and $5 \mathrm{~A}$ ), course (Figures 4D and 5A) and branching pattern (Figures 4B and 5B) of aorta and pulmonary artery trunk. Regarding the veno-atrial connections (Figure 4, A and B), it was easy to identify the entrance of the inferior and superior cava veins into the right atrium, but a detailed review of the histological sections was necessary for a confident visualization of all left atrium venous openings.
We noted a lower resolution or quality of the "reconstructed" planes, mainly caused by the inherent morphological deformation following heart block sectioning. However, these distortions did not represent an impediment to visualize the heart anatomy structures in any of the five fetuses.
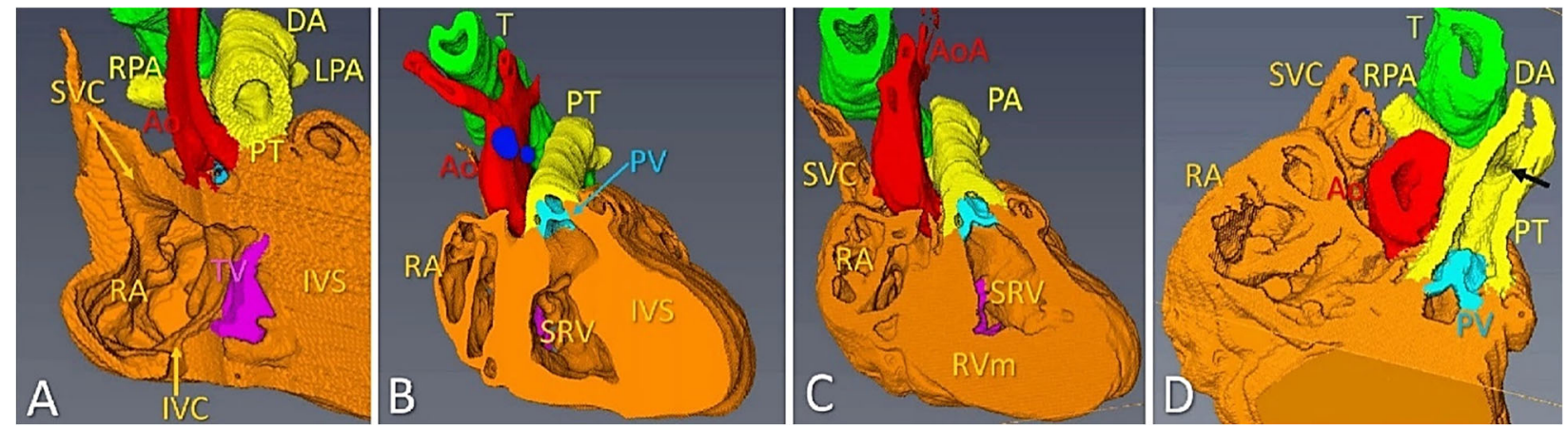

Figure 4 - Right heart evaluation: (A) Bicaval plane, showing the SVC and IVC atrial connections, indicated with arrows; (B) RVOT with PV highlighted at the emergence of PT from the right ventricle and intact adjacent septum; (C) Rotation of the fetal heart to visualize the rest (anterior part) of the IVS; (D) PT short axis view with trifurcation of the main PA into the RPA, which wraps around the arta, the LPA (indicated with arrow), and the large DA. Ao: Aorta; AoA: Aortic arch; DA: Ductus arteriosus; IVC: Inferior vena cava; IVS: Interventricular septum; LPA: Left pulmonary artery; PA: Pulmonary artery; PT: Pulmonary trunk; PV: Pulmonary valve; RA: Right atrium; RPA: Right pulmonary artery; $R V m$ : Right ventricle myocardium; RVOT: Right ventricular outflow tract; SRV: IVS view from the right ventricle; SVC: Superior vena cava; T: Trachea; TV: Tricuspid valve.
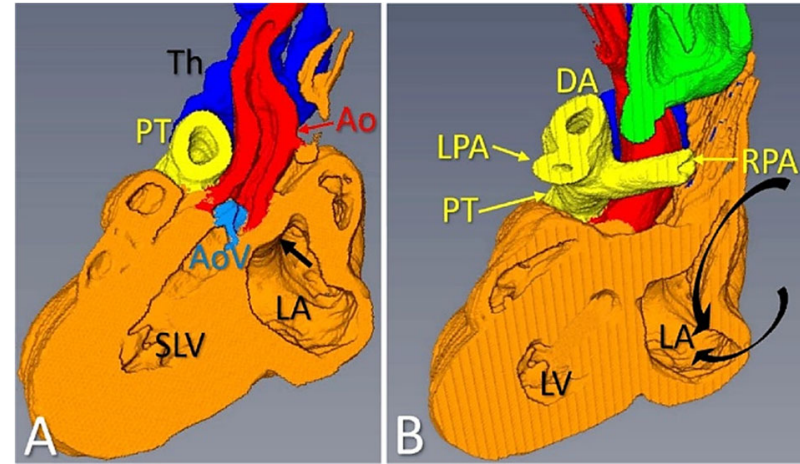

Figure 5 - Left heart evaluation: (A) IVS view from the LV and LVOT (aortic emergence) with AoV highlighted in blue - interatrial communication view from the $L A$, indicated with arrow; (B) Two venous connections of the LA (black curved arrows) - concomitantly, pulmonary artery trifurcation is better visualized. Ao: Aorta; AoV: Aortic valve; DA: Ductus arteriosus; IVS: Interventricular septum; LA: Left atrium; LPA: Left pulmonary artery; LV: Left ventricle; LVOT: Left ventricular outflow tract; PT: Pulmonary trunk; RPA: Right pulmonary artery; SLV: IVS view from the left ventricle; Th: Thymus.

\section{Discussions}

We present a protocol of standard histological preparation, which ultimately enables the virtual reconstruction and analysis of fetal cardiac structures at the time of first trimester anomaly scan, 11-13 GW. This is important, because the fetal heart's size over the span of the first trimester anomaly scan generates an important dilemma regarding the best method to audit the heart structures normalcy in pregnancy termination cases. At this age, the fetal heart is too small for standard pathology investigation

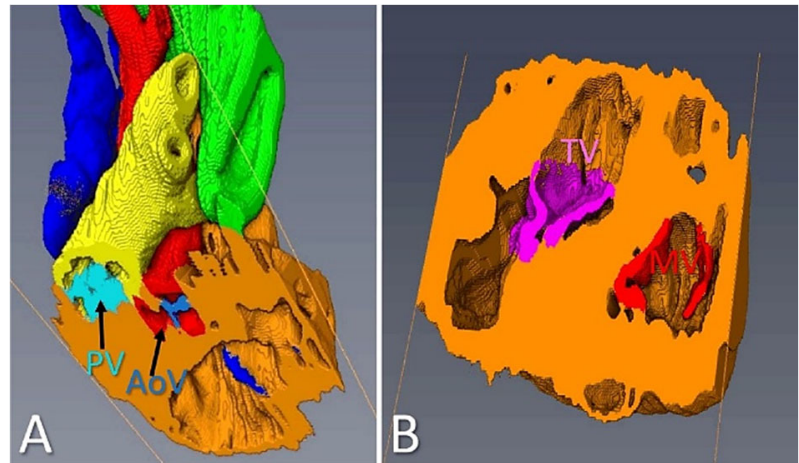

Figure 6 - Heart section showing the valves of the great vessels (A) and AV valves (B) in the same plane. AoV: Aortic valve; AV: Atrioventricular; MV: Mitral valve; PV: Pulmonary valve; TV: Tricuspid valve.

methods [24] and conventional MRI [11-13], too big for alternate imaging methods, as the photonic confocal microscopy [20, 25-27], US backscatter microscopy [28, 29], and optical coherence tomography (OCT) [30-32], while non-invasive high-resolution imaging techniques, as 7-9.4 T MRI [6-8] and micro-CT [9, 10] are still performed only in research settings due to the low availability and limited data regarding the reliability of first trimester heart anomalies detection. The principle of 3D reconstructions of embryonic organs based on computer-aided techniques is not new $[16,19]$ and in our opinion, should not remain a historical one, but improved for the first trimester autopsy purposes. In our experience, the use of the technical advances in histological preparation and computer-imaging software overcome the previous highlighted disadvantages of the method. Thus, we developed a protocol that involves basic resources for general settings, to obtain an accurate and reproducible HP imaging of the first trimester fetal heart. 
The results of our pilot study showed that the main features of the fetal heart anatomy, including atria and ventricles features, septum, and ventricle outflow tracts emergence and branching were achievable in all cases using low resources in terms of equipment and medical personnel. It was much harder to highlight left atrium venous inflows, but the need to confirm abnormalities of these structures is negligible in the first trimester, as the veins identification is not part of the routinely US scan protocol.

Non-invasive imaging tools were proposed to serve as audit for first trimester anomaly, especially in the context of alarmingly decreasing rates of fetal conventional autopsy. In our view, it is questionable to audit an imaging in vivo technique (US) with another one (MRI, CT) performed postmortem. Poor image resolution of conventional MRI accounts for significant errors regarding heart malformations detection in fetuses less than $24 \mathrm{GW}[7,11,13]$. This problem of image resolution in small-sized fetuses could be addressed by carrying out postmortem MRI at high-field strength or CT angiography $[7,9]$. By utilizing high-field postmortem MRI at 9.4 T, the four-chamber view and outflow tracts can be viewed in all fetuses irrespective of gestational age (8 GW), while micro-CT improved resolution for imaging small objects down to micrometers. Still, the human and technical resources for such investigation are lacking in general settings. This is also the main disadvantage of advanced pathology techniques, such as episcopic microscopy.

The literature and our studies as well highlighted the need for an accurate early diagnosis in pregnancy [33-38]. Therefore, the main objective of our research was to develop a feasible method to imagine the fetal heart in the first trimester. As various techniques were proposed, we aimed to use an optimal technique in terms of resources involved, such as medical equipment requirements, software, personnel, and time. 3D reconstruction of histological sections based on computer-aided manual tracing appears the optimal alternative for the conventional autopsy for several reasons. First, the requirements for the protocol are very lenient. Then, the use of histology offers the possibility to reanalyze the original heart block and correctly identify the tissue characteristics. It is also important that any sectional planes may be reconstructed to imagine various heart structures that may be considered important for the respective case.

Regarding the resources we used, no custom-made hardware is necessary: stained sections were digitized using an ordinary scanner and computer. The other methods we mentioned as a replacement for conventional autopsy require professional, dedicated, and expensive equipment and software. The software used for the reconstruction procedure does not require programming skills and was designed for end users and can be purchased at a reasonable amount of money. To further decrease the time involved by the protocol, many steps may be automated: cutting, coloring, and scanning.

Our work represents a pilot project that confirms the viability of cardiac structures reconstruction from histological sections in first trimester fetuses. The results denote that this method can be applied to regular clinical practice. The method we used has several strong points.
The image reconstruction resolution from histological sections is higher than conventional imaging methods, while the heart volume information can be reexplored using two-dimensional (2D) and 3D imaging.

\section{Study limitation}

We acknowledge the preliminary nature of this pilot study, study which is limited to the reconstruction of several normal hearts. The aim of our research was to emphasis the applicability of the method in nowadays settings. However, the quality of the images we have obtained leaves little doubt that the method will be feasible for the study of abnormal hearts.

Histological investigation requires a conventional autopsy, one that has a much lower acceptance by parents than non-invasive virtuopsy, which is accepted by nearly all mothers. Thus, the high acceptance of virtuopsy makes it a more acceptable alternative, as the rate of refusal of conventional autopsy is rising and usually depends on factors over which we have no control [39].

A lower resolution of the "reconstructed" planes was noted compared with the other imaging methods. This morphological deformation, inherent during heart block sectioning and preparation could be corrected using external marker-based automatic congruency [40], although this approach was previously challenged because of the difficulties to place external markers close enough to the fetal heart [20]. This field may benefit from implementation of image processing solutions that improve modeling of distortions. Such correction software solutions are used in other imaging methods, as micro-MRI or US.

\section{ㅁ Conclusions}

The use of 3D reconstruction of fetal heart histological sections in first trimester has an important potential to confirm heart anatomy, even in this era of great advances in microscopy and imaging techniques. This method can be used during routine clinical practice with few and low-cost supplementary resources. Image reconstruction from histological sections offers an excellent resolution while all the information is retained, and the volume can be stored, reexplored or sent online for a second opinion. The procedure would benefit from refinements used in other imaging techniques to limit human-computer interactions, such as sections distortion.

\section{Conflict of interests}

The authors declare that they have no conflict of interests.

\section{Acknowledgments}

Microscopic images have been acquired in the Research Center for Microscopic Morphology and Immunology, University of Medicine and Pharmacy of Craiova, Romania (Manager: Laurenţiu Mogoantă, Professor, MD, PhD).

Authors' contribution

Dan Ruican and Ana-Maria Petrescu equally contributed to this article.

\section{References}

[1] Nicolaides $\mathrm{KH}$. A model for a new pyramid of prenatal care based on the 11 to 13 weeks' assessment. Prenat Diagn, 2011, 31(1):3-6. https://doi.org/10.1002/pd.2685 PMID: 21210474 
[2] Salomon LJ, Alfirevic Z, Bilardo CM, Chalouhi GE, Ghi T, Kagan KO, Lau TK, Papageorghiou AT, Raine-Fenning NJ, Stirnemann J, Suresh S, Tabor A, Timor-Tritsch IE, Toi A Yeo G. ISUOG practice guidelines: performance of first-trimester fetal ultrasound scan. Ultrasound Obstet Gynecol, 2013, 41(1): 102-113. https://doi.org/10.1002/uog.12342 PMID: 23280739

[3] Coady AM, Bower S (eds). Twining's textbook of fetal abnormalities. First-trimester detection of fetal anomalies. $3^{\text {rd }}$ edition, Churchill Livingstone-Elsevier, London, UK, 2014 , 58-79. https://www.elsevier.com/books/twinings-textbook-offetal-abnormalities/9780702045912

[4] Vogt C, Blaas HGK, Salvesen KÄ, Eik-Nes SH. Comparison between prenatal ultrasound and postmortem findings in fetuses and infants with developmental anomalies. Ultrasound Obstet Gynecol, 2012, 39(6):666-672. https://doi.org/10.1002/uog. 10106 PMID: 21953982

[5] Şorop-Florea M, Ciurea RN, loana M, Stepan AE, Stoica GA Tănase $F$, Comănescu $M C$, Novac MB, Drăgan I, Pătru $C L$, Drăguşin RC, Zorilă GL, Cărbunaru OM, Oprescu ND, Ceauşu I, Vlădăreanu S, Tudorache S, lliescu DG. The importance of perinatal autopsy. Review of the literature and series of cases. Rom J Morphol Embryol, 2017, 58(2):323-337. PMID: 28730216

[6] Staicu A, Albu C, Popa-Stanila R, Chiriac L, Boitor-Borza D, Bondor C, Kovacs T, Caracostea G, Rotar IC, Turcu RVF, Simon S, Muresan D, Stamatian F. Potential clinical benefits and limitations of fetal virtopsy using high-field MRI at 7 Tesla versus stereomicroscopic autopsy to assess first trimester fetuses. Prenat Diagn, 2019, 39(7):505-518. https://doi.org/ 10.1002/pd.5457 PMID: 30980413

[7] Thayyil S, Cleary JO, Sebire NJ, Scott RJ, Chong K, Gunny R, Owens CM, Olsen OE, Offiah AC, Parks HG, Chitty LS, Price AN, Yousry TA, Robertson NJ, Lythgoe MF, Taylor AM. Post-mortem examination of human fetuses: a comparison of whole-body high-field MRI at $9.4 \mathrm{~T}$ with conventional MRI and invasive autopsy. Lancet, 2009, 374(9688):467-475. https:// doi.org/10.1016/S0140-6736(09)60913-2 PMID: 19665645

[8] Votino C, Jani J, Verhoye M, Bessieres B, Fierens Y, Segers V, Vorsselmans A, Kang X, Cos T, Foulon W, De Mey J, Cannie M. Postmortem examination of human fetal hearts at or below 20 weeks' gestation: a comparison of high-field MRI at 9.4 T with lower-field MRI magnets and stereomicroscopic autopsy. Ultrasound Obstet Gynecol, 2012, 40(4):437-444. https://doi. org/10.1002/uog.11191 PMID: 22605566

[9] Lombardi CM, Zambelli V, Botta G, Moltrasio F, Cattoretti G, Lucchini V, Fesslova V, Cuttin MS. Postmortem microcomputed tomography (micro-CT) of small fetuses and hearts. Ultrasound Obstet Gynecol, 2014, 44(5):600-609. https://doi.org/10.1002/ uog.13330 PMID: 24585450

[10] Hutchinson JC, Arthurs OJ, Ashworth MT, Ramsey AT, Mifsud W, Lombardi CM, Sebire NJ. Clinical utility of postmortem microcomputed tomography of the fetal heart: diagnostic imaging vs macroscopic dissection. Ultrasound Obstet Gynecol, 2016 , 47(1):58-64. https://doi.org/10.1002/uog.15764 PMID: 26415141

[11] Thayyil S, Sebire NJ, Chitty LS, Wade A, Olsen O, Gunny RS Offiah A, Saunders DE, Owens CM, 'Kling' Chong WK, Robertson NJ, Taylor AM. Post mortem magnetic resonance imaging in the fetus, infant and child: a comparative study with conventional autopsy (MaRIAS Protocol). BMC Pediatr, 2011, 11:120. https://doi.org/10.1186/1471-2431-11-120 PMID: 22192497 PMCID: PMC3259035

[12] Sandaite I, Dymarkowski S, De Catte L, Moerman P, Gewillig M, Fedele L, Deprest J, Claus F. Fetal heart pathology on postmortem 3-T magnetic resonance imaging. Prenat Diagn, 2014, 34(3):223-229. https://doi.org/10.1002/pd.4283 PMID: 24243492

[13] Taylor AM, Sebire NJ, Ashworth MT, Schievano S, Scott RJ Wade A, Chitty LS, Robertson N, Thayyil S; Magnetic Resonance Imaging Autopsy Study Collaborative Group. Postmortem cardiovascular magnetic resonance imaging in fetuses and children: a masked comparison study with conventional autopsy. Circulation, 2014, 129(19):1937-1944. https://doi.org/10.1161/ CIRCULATIONAHA.113.005641 PMID: 24647275

[14] Matsui H, Mohun T, Gardiner HM. Three-dimensional reconstruction imaging of the human foetal heart in the first trimester. Eur Heart J, 2010, 31(4):415. https://doi.org/10.1093/eurheartj/ ehp511 PMID: 19965862

[15] Gindes L, Matsui H, Achiron R, Mohun T, Ho SY, Gardiner H. Comparison of ex-vivo high-resolution episcopic microscopy with in-vivo four-dimensional high-resolution transvaginal sonography of the first-trimester fetal heart. Ultrasound Obstet Gynecol, 2012, 39(2):196-202. https://doi.org/10.1002/uog. 9068 PMID: 21638368

[16] Verbeek FJ, Huijsmans DP, Baeten RJ, Schoutsen NJ, Lamers WH. Design and implementation of a database and program for 3D reconstruction from serial sections: a datadriven approach. Microsc Res Tech, 1995, 30(6):496-512. https://doi.org/10.1002/jemt.1070300607 PMID: 7599361

[17] Weninger WJ, Mohun T. Phenotyping transgenic embryos: a rapid 3-D screening method based on episcopic fluorescence image capturing. Nat Genet, 2002, (1):59-65. https://doi.org/ 10.1038/ng785 PMID: 11743576

[18] Baldock RA, Verbeek FJ, Vonesch JL. 3-D Reconstructions for graphical databases of gene expression. Semin Cell Dev Biol, 1997, 8(5):499-507. https://doi.org/10.1006/scdb.1997. 0175 PMID: 9441955

[19] Huijsmans DP, Lamers WH, Los JA, Strackee J. Toward computerized morphometric facilities: a review of 58 software packages for computer-aided three-dimensional reconstruction, quantification, and picture generation from parallel serial sections. Anat Rec, 1986, 216(4):449-470. https://doi.org/10.1002/ar. 1092160402 PMID: 3541684

[20] Soufan AT, Ruijter JM, van den Hoff MJB, de Boer PAJ, Hagoort J, Moorman AFM. Three-dimensional reconstruction of gene expression patterns during cardiac development. Physiol Genomics, 2003, 13(3):187-195. https://doi.org/10. 1152/physiolgenomics.00182.2002 PMID: 12746463

[21] Schleich JM, Dillenseger JL, Loeuillet L, Moulinoux JP, Almange $\mathrm{C}$. Three-dimensional reconstruction and morphologic measurements of human embryonic hearts: a new diagnostic and quantitative method applicable to fetuses younger than 13 weeks of gestation. Pediatr Dev Pathol, 2005, 8(4):463-473. https://doi.org/10.1007/s10024-005-0017-8 PMID: 16211458 PMCID: PMC2104785

[22] Belciug S. Artificial intelligence in cancer: diagnostic to tailored treatment. $1^{\text {st }}$ edition, Elsevier-Academic Press, San Diego, USA, 2020. https://doi.org/10.1016/C2019-0-00740-6

[23] Şerbănescu MS, Pleşea IE. A hardware approach for histological and histopathological digital image stain normalization. Rom J Morphol Embryol, 2015, 56(2 Suppl):735-741. PMID: 26429166

[24] Isaksen CV, Eik-Nes SH, Blaas HG, Tegnander E, Torp SH. Comparison of prenatal ultrasound and postmortem findings in fetuses and infants with congenital heart defects. Ultrasound Obstet Gynecol, 1999, 13(2):117-126. https://doi.org/10.1046/ j.1469-0705.1999.13020117.x PMID: 10079491

[25] Rodenacker K, Hausner M, Kühn M, Wuertz S, Purkayastha S. Depth intensity correction of biofilm volume data from confocal laser scanning microscopes. Proceedings of the $8^{\text {th }}$ European Congress for Stereology (ECS) and Image Analysis, September 4-7, 2001 Bordeaux, France, Image Anal Stereol, 2001, 20(Suppl 1):556-560. https://citeseerx.ist.psu.edu/viewdoc/ download?doi=10.1.1.9.220\&rep=rep1\&type=pdf

[26] Hecksher-Sørensen J, Sharpe J. 3D confocal reconstruction of gene expression in mouse. Mech Dev, 2001, 100(1):5963. https://doi.org/10.1016/s0925-4773(00)00508-6 PMID: 11118884

[27] Verbeek FJ, Lawson KA, Bard JB. Developmental bioinformatics: linking genetic data to virtual embryos. Int J Dev Biol, 1999, 43(7):761-771. PMID: 10668984

[28] Turnbull DH. In utero ultrasound backscatter microscopy of early stage mouse embryos. Comput Med Imaging Graph, 1999, 23(1):25-31. https://doi.org/10.1016/s0895-6111(98)0 0060-3 PMID: 10091865

[29] Srinivasan S, Baldwin HS, Aristizabal O, Kwee L, Labow M, Artman $\mathrm{M}$, Turnbull $\mathrm{DH}$. Noninvasive, in utero imaging of mouse embryonic heart development with $40-\mathrm{MHz}$ echocardiography. Circulation, 1998, 98(9):912-918. https://doi.org/10.1161/01. cir.98.9.912 PMID: 9738647

[30] Boppart SA, Brezinski ME, Fujimoto JG. Optical coherence tomography imaging in developmental biology. Methods $\mathrm{Mol}$ Biol, 2000, 135:217-233. https://doi.org/10.1385/1-59259685-1:217 PMID: 10791319

[31] Gupta M, Rollins AM, Izatt JA, Efimov IR. Imaging of the atrioventricular node using optical coherence tomography. J Cardiovasc Electrophysiol, 2002, 13(1):95. https://doi.org/ 10.1046/j.1540-8167.2002.00095.x PMID: 11843494 
[32] Yelbuz TM, Choma MA, Thrane L, Kirby ML, Izatt JA. Optical coherence tomography: a new high-resolution imaging technology to study cardiac development in chick embryos. Circulation, 2002, 106(22):2771-2774. https://doi.org/10.1161/ 01.cir.0000042672.51054.7b PMID: 12451001

[33] Gheorman L, Iliescu D, Ceauşu I, Paulescu D, Pleşea IE, Gheorman V. Importance of early complex evaluation in high-risk pregnancy associated to diabetes mellitus. Case presentation and review of the literature. Rom $\mathrm{J}$ Morphol Embryol, 2011, 52(3 Suppl):1127-1132. PMID: 22119836

[34] Iliescu D, Tudorache S, Comanescu A, Antsaklis P, Cotarcea S, Novac L, Cernea N, Antsaklis A. Improved detection rate of structural abnormalities in the first trimester using an extended examination protocol. Ultrasound Obstet Gynecol, 2013, 42(3):300-309. https://doi.org/10.1002/uog.12489 PMID: 23595897

[35] Iliescu DG, Comanescu AC, Tudorache S, Cernea N. Right aortic arch with patent right ductus arteriosus and normal heart Ultrasound Obstet Gynecol, 2012, 40(1):115-116. https://doi. org/10.1002/uog.10076 PMID: 21858884

[36] Iliescu D, Comănescu A, Antsaklis P, Tudorache S, Ghiluşi M, Comănescu V, Paulescu D, Ceauşu I, Antsaklis A, Novac L, Cernea N. Neuroimaging parameters in early open spina bifida detection. Further benefit in first trimester screening? Rom J Morphol Embryol, 2011, 52(3):809-817. PMID: 21892523
[37] Iliescu DG, Cara ML, Tudorache S, Antsaklis P, Novac LV, Antsaklis A, Cernea N. Agenesis of ductus venosus in sequential first and second trimester screening. Prenat Diagn, 2014, 34(11):1099-1105. https://doi.org/10.1002/pd.4434 PMID: 24931552

[38] Tudorache S, Cara M, lliescu DG, Novac L, Cernea N. First trimester two- and four-dimensional cardiac scan: intra- and interobserver agreement, comparison between methods and benefits of color Doppler technique. Ultrasound Obstet Gynecol, 2013, 42(6):659-668. https://doi.org/10.1002/uog.12459 PMID: 23494803

[39] Cannie M, Votino C, Moerman Ph, Vanheste R, Segers V, Van Berkel K, Hanssens M, Kang X, Cos T, Kir M, Balepa L, Divano L, Foulon W, De Mey J, Jani J. Acceptance, reliability and confidence of diagnosis of fetal and neonatal virtuopsy compared with conventional autopsy: a prospective study. Ultrasound Obstet Gynecol, 2012, 39(6):659-665. https:// doi.org/10.1002/uog.10079 PMID: 21919100

[40] Streicher J, Donat MA, Strauss B, Spörle R, Schughart K, Müller GB. Computer-based three-dimensional visualization of developmental gene expression. Nat Genet, 2000, 25(2): 147-152. https://doi.org/10.1038/75989 PMID: 10835627

\section{Corresponding authors}

Anda Laura Ungureanu, MD, Department of Cardiology, University of Medicine and Pharmacy of Craiova, 2 Petru Rareş Street, 200349 Craiova, Dolj County, Romania; Phone +40799-995 257, e-mail: ungureanu_anda@yahoo.com Mircea-Sebastian Şerbănescu, Lecturer, MD, PhD, Department of Medical Informatics and Biostatistics, University of Medicine and Pharmacy of Craiova, 2 Petru Rareş Street, 200349 Craiova, Dolj County, Romania; Phone +40745-766 610, e-mail: mircea_serbanescu@yahoo.com 